\title{
Reduced Field-driven Domain Wall Injection Field In Permalloys Nanowires Obtained by Destroying Symmetry
}

\author{
Hong-Yo Lu, Kuei-Chang Hu, Feng-Sheng Wu, \\ Yee-Mou Kao, Jong-Ching Wu and Lance Horng* \\ Department of Physics, National Changhua University of Education, Changhua 500, Taiwan
}

\begin{abstract}
Domain wall motion in Permalloy nanowires has been extensively investigated, because of its potential application in new types of memory devices. We injected a domain wall into permalloy nanowires to observe domain wall motion in the nanowires, and we observed the injection behavior from an extended injection pad by using a Landau-Lifshitz-Gilbert simulator (LLG) based on LLG equation. We found that the injection field was reduced by destroying the symmetry of the Permalloy nanowires. Specifically, the injection field reduced from 195 Oe to 60 Oe by shifting the nanowires away from the center of the disk and cutting away the top of the disk.
\end{abstract}

Key words: Field-driven, injection, nanowires, destruction of symmetry

\section{Introduction}

Base on the new concept, Parkin et.al envisioned an innovative new concept of memory storage device "Racetrack memory"1), which is different from any other memory device now existing. Racetrack memory device is a $3 \mathrm{D}$ structure device, with high memory storage density and composed of numerous nanowires and magnetic tunneling junction (MTJ) sensing devices. For the magnetic domains to enter the nanowire, a highly spin-polarized current was injected to create the magnetic domains and move them along the Permalloy nanowires. ${ }^{2)}$ In addition to the current-induced domain wall motion, we can also applying an external field parallel to the nanowires to drive the domain walls. ${ }^{3)}$ In this article, we use the LLG simulator based on Landau-Lifshitz-Gilbert (LLG) equation to investigate the field-driven domain-wall injection field in the nanowire. ${ }^{4)}$ ) The injection field of 195 Oe is applied to inject a domain wall from the extended disk into the long, straight Permalloy nanowire. In this simulation, we found that we can reduce the field-driven domain-wall injection field significantly via destroying the symmetry of nanowires with disk-shaped pad. The symmetry was destroyed by the offset nanowire and by cutting away a part of the disk as schematically illustrated in Fig. 1 and by both of the methods the injection field was reduced from 195 Oe to 40 Oe.

\section{Simulation}

Simulations were performed with the LLG simulator., which is based on Landau-Lifshitz-Gilbert (LLG) equation. ${ }^{6)}$ To investigate the domain wall injection field from the extended disk into the nanowire, a long straight nanowire with disk-shaped pad was designed as schematically illustrated in Fig. 1. The nanowire is $3000 \mathrm{~nm}$ long, $100 \mathrm{~nm}$ wide, and 10nm thick; the disk has a radius of $500 \mathrm{~nm}$. In order to induce the domain wall into the system, the disk-shaped pad was located on the left end of the nanowire. The parameters for permalloy are the magnetization $\mathrm{M}_{\mathrm{s}}=8.0 \times 10^{5} \mathrm{~A} / \mathrm{m}$, exchange stiffness $\mathrm{A}=1.3 \times 10^{-11} \mathrm{~J} / \mathrm{m}$, alpha $\alpha=0.008$, gamma $\mathrm{Y}=$ 17.6 MHz/Oe sec, zero magnetocrystalline anisotropy and cell size $5 \times 5 \times 5 \mathrm{~nm}^{3}$. And we use different methods to destroy the symmetry of the nanowire with disk-shaped pad as the schematically illustrated in Fig. 2. In this simulation, we observe the relationship between the destruction extent and the injection field. We set the magnetic moment in $-x$ direction , and we applied a positive field parallel to the nanowire from -1000 Oe to 0 Oe, and we see it as the initial state. And then applied a positive constant injection field parallel to the nanowire. When the domain wall go through from the extended injection pad to the nanowire, we can set it as the domain wall's injection field.

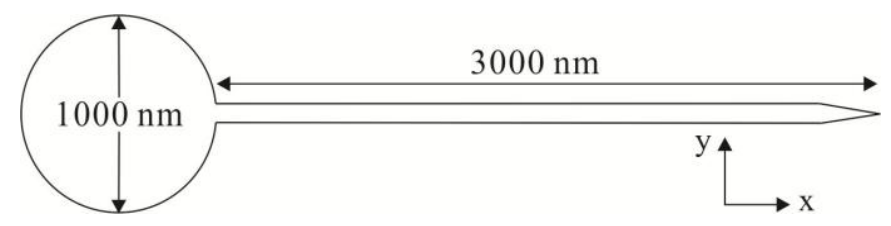

Fig. 1 Schematic of the nanowire with disk. Diameter of nanodisk is $1000 \mathrm{~nm}$ and its cross is $100 \mathrm{x} 10 \mathrm{~nm}$. 


\section{Result and Discussion}

We destroyed the symmetry of the nanowire with disk-shaped pad with two methods. In the first method the nanowire was shifted from the center of the disk to the top with a distance ranging from $0 \mathrm{~nm}$ to $450 \mathrm{~nm}$. In the second method the top of the disk was cut away with different angle $\theta$ from 0 degree to 84 degrees. These two methods reduced the injection field significantly as shown in Fig. 3. The symmetry was destroyed by shifting the nanowire, and the minimal injection field was obtained when the shift distance was $S=200,300$, $400 \mathrm{~nm}$ and the injection field was 60 Oe. Although the injection field were the same, the injection time were the fastest when the shift distance $\mathrm{S}=200 \mathrm{~nm}$. The domain wall moved more displacement when the shift distance increasing from $200 \mathrm{~nm}$ to $400 \mathrm{~nm}$, and the injection time also increased. By cutting away the top of the disk with different angles, the minimal injection field was investigated when the angle $\theta=60$ degree and the injection field was 40 Oe. We confirmed that, destroying the symmetry reduced the injection field significantly and the optimal way was to cutting away the top of disk with an cut away angle $\theta=60$ degrees. And the injection field rose when the cut away angle $\theta$ $=84$ degrees and the shift distance was $\mathrm{S}=450 \mathrm{~nm}$ as shown in Fig 4. We conjecture the sharp side of the nanowires might affect the injection behavior of the domain wall. The sharp side made the magnetic moment arrangement with the shape and it caused the stray field at this place. And the stray field will let the domain wall's injection field to rise when the cut away angle $\theta=84$ degrees and the shift distance was $\mathrm{S}=$ $450 \mathrm{~nm}$.

(a)

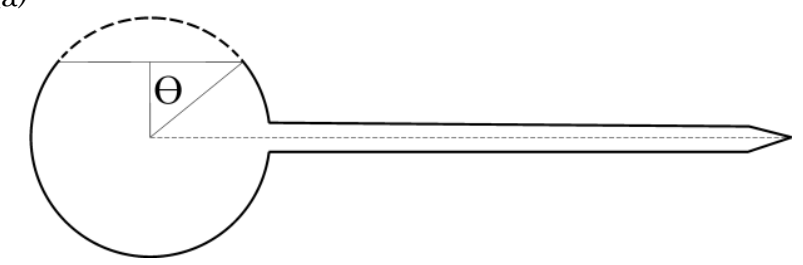

(b)

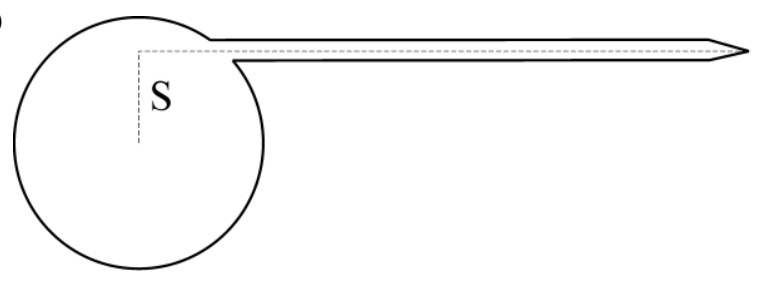

Fig. 2 Different methods were used to destroy the symmetry of nanowires with disk-shaped pads.

One method shown in (a) was to excise the top of disk, and another one shown in (b) was to shift nanowire away to the center of disk (a)
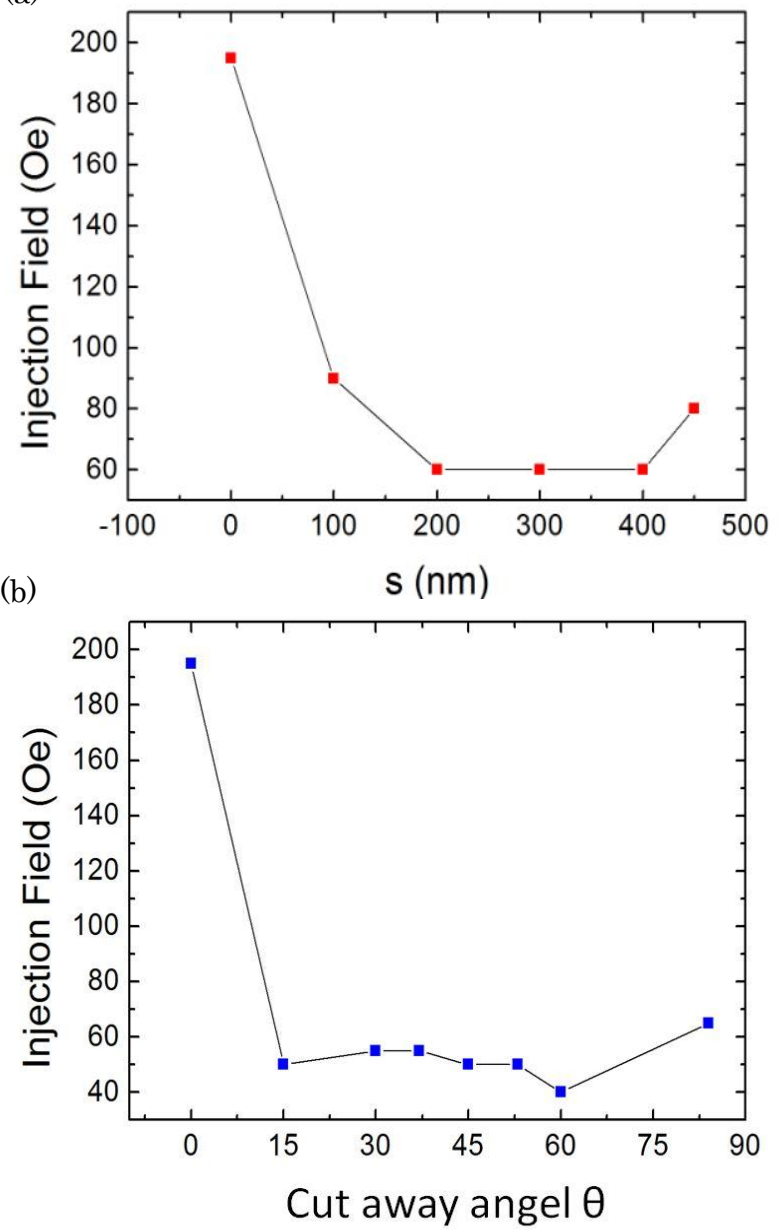

(c)

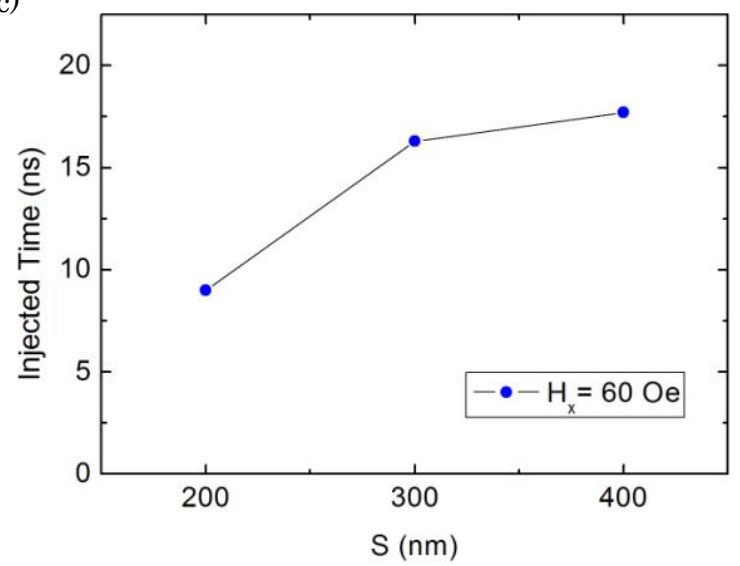

Fig. 3 (a) showed the injection field as function of different displacement of a nanowire that shifted from the center of the disk from $0 \mathrm{~nm}$ to $450 \mathrm{~nm}$. (b) showed the injection field as a function of cut away angle $\theta$ from 0 degree to 84 degrees. (c) showed the injection time as a function of displacements 200, 300, and $400 \mathrm{~nm}$. In the same injection field, it was found that the injection time was the shortest when the nanowire was 
(a)

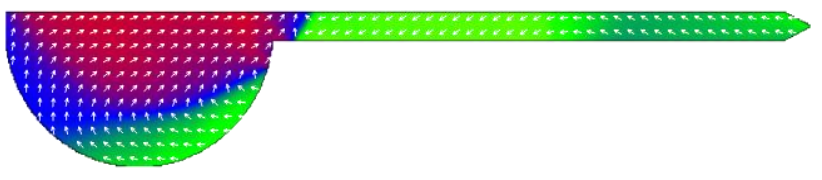

(b)

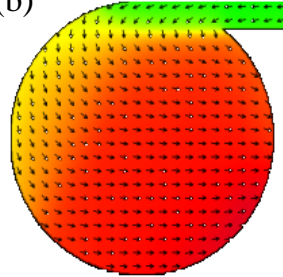

Fig. 4 (a) showed the magnetic moment distribution in the nanowire shifting from the center of the disk $450 \mathrm{~nm}$. (b) showed the magnetic moment distribution in the nanowire with cutting away angle 84 degrees.

\section{Conclusion}

In conclusion, injecting the domain wall to the nanowire from the extended nanodisk that was located on one end of the nanowire is demonstrated and the optimal way to reduce the injection field is to cut away the top of the disk-shaped injection pad. The injection field was reduced from 195 Oe to 40 Oe. We can reduce the injection field easily by destroying the symmetry of the nanowires.

\section{Reference}

1) S. S. P. Parkin, M. Hayashi, and L. Thomas, "Magnetic domain-wall racetrack memory," Science, vol. 320, pp.190-194, (2008).

2) J. H. Ai, B. F. Miao, L. Sun, B. You, An Hu, and H. F. Ding, "Current-induced domain wall motion in permalloy nanowires with a rectangular cross-section," J. Appl. Phys. vol. 110, 093913, (2011).

3) M. Hayashi, L. Thomas,1 Ya. B. Bazaliy, C. Rettner, R. Moriya, X. Jiang, and S. S. P. Parkin, "Influence of Current on Field-Driven DomainWall Motion in Permalloy Nanowires from Time Resolved Measurements of Anisotropic Magnetoresistance," Phys. Rev. Lett, vol. 96, 197207, (2006).

4) N. L. Schryer and L. R. Walker, "The motion of $180^{\circ}$ domain walls in uniform dc magnetic fields," J. Appl. Phys. 45, 5406, (1974).

5) F. Bloch, "Zur Theorie des Austauschproblems under Remanenzer-scheinung der Ferromagnetika," Z. Phys. 74, 295, (1932)

6) M. R. Scheinfein, LLG Micromagnetic Simulator ${ }^{\mathrm{TM}}$ http://llgmicro.home.mindsrping.com.

along thin, narrow strips," J. Appl. Phys., vol. 95, p. 6729, (2004).

Received Dec. 16, 2013; Revised Feb. 05, 2014; Accepted Sep. 02,2014 